\title{
Compact Optical Waveguides Based on Hybrid Index and Surface-Plasmon-Polariton Guidance Mechanisms
}

\author{
Min Yan and Min Qiu \\ Department of Microelectronics and Applied Physics, Royal Institute of Technology, Electrum 229, 16440 Kista, Sweden
}

Received 13 October 2007; Accepted 9 November 2007

Recommended by Yalin Lu

Surface-plasmon-polariton (SPP) waveguides made of materials available in nature have, in general, been found to suffer from very high absorption loss when light confinement is beyond diffraction limit. In this paper, the possibility of combining both the conventional index-guiding and the SPP-guiding mechanisms together into one single waveguide is being explored. Such waveguides, expectedly, inherent the low-loss feature of all-dielectric waveguides as well as the superior mode field confinement possessed by SPP waveguides. By using experimentally ready materials, it is theoretically shown that compact metallodielectric waveguides can be designed with a $\sim 500 \times 500 \mathrm{~nm}^{2}$ core size around the $1550 \mathrm{~nm}$ telecommunication wavelength. The examined waveguides can be interpreted as a gap SPP waveguide with an inner dielectric core. Compared to pure SPP waveguides, such hybrid waveguides have a comparable mode field size, but with significantly lower loss $(\sim 0.05 \mathrm{~dB} / \mu \mathrm{m}$ for either quasi-TE or quasi-TM operation). Therefore they can be potentially deployed for a range of integrated photonic applications.

Copyright (C) 2007 M. Yan and M. Qiu. This is an open access article distributed under the Creative Commons Attribution License, which permits unrestricted use, distribution, and reproduction in any medium, provided the original work is properly cited.

\section{INTRODUCTION}

Recently, there has been a revived interest on surfaceplasmon-polariton (SPP) waveguides [1]. Such waveguides can confine light without a diffraction limit. Therefore, they are promising for applications in integrated optics. However, the propagation loss of almost all SPP waveguides is found to be inversely proportional to their mode field confinement (see, e.g., [2]). Most SPP waveguides proposed so far have their propagation lengths limited to only several micrometers once light is squeezed beyond the diffraction limit [35]. Such a high loss value ultimately prevents the waveguides from being deployed as integrated photonic circuits. In this paper, we look into a type of waveguides which guide light using both the dielectric index-guiding mechanism and the SPP effect. Such hybrid waveguides should inherit both the low-loss feature of all-dielectric waveguides as well as the superior mode confinement possessed by SPP waveguides. Compared to SPP waveguides, the proposed waveguides are expected to be able to achieve a comparable mode field confinement and lower propagation loss.

The waveguide to be examined, in a 1D slab limit, has a high-index dielectric core layer surrounded by low-index dielectric cladding layers, which is then further jacketed by metal claddings (see Figure 1(a)). When the distance be- tween two metals (or metal gap size) is large enough, both TE and TM modes can be guided by the central high-index dielectric core. As the gap distance gets smaller, the effect of metal claddings becomes more evident. In fact, if the gap distance is close to the wavelength, the dielectric materials between metal claddings can be treated as an effective dielectric medium. Among the two modes, the TE mode will be cutoff if the metal gap is as short as half of the light wavelength (measured in the effective dielectric medium), while the TM mode is an SPP in nature and does not suffer from a diffraction limit. In practice, the waveguide in Figure 1(a) should be fabricated with a finite height. Figure 1(b) depicts the schematic cross-section of a corresponding planar 2D waveguide. Modes guided by the waveguide shown in Figure 1(b) are all hybrid. The first two modes have their major electric field polarized along either $x$ or $y$ direction. These two modes, therefore, can be referred to as quasi-TE or quasi-TM mode. Both index-guiding and SPP-guiding mechanisms, in general, contribute to the mode field confinement in such a waveguide. Without the central high-index core, the waveguides shown in Figures 1(a) and 1(b) correspond to the previously reported metal-insulator-metal (MIM) [6] and metal gap waveguides $[4,7-9]$, respectively. We will show that introduction of a high-index inner core will reduce metal absorption loss considerably for the TM mode (or quasi-TM 


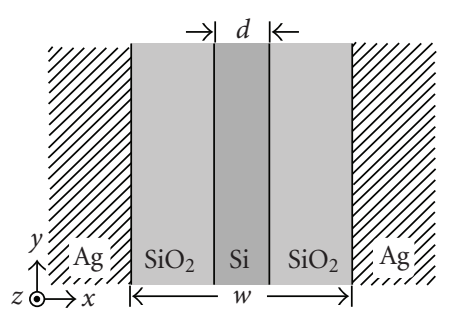

(a)

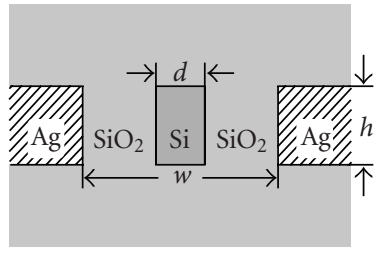

(b)
FIgURE 1: Schematic diagrams of the hybrid optical waveguide cross-sections. (a) 1D; (b) 2D.

mode for a $2 \mathrm{D}$ waveguide). At the same time, the TE mode (or quasi-TE mode) can be saved from cutoff, due to an increase in the effective index of the dielectric filling between two metal claddings. The optimum inner core thickness will be determined for achieving relatively low-loss quasi-TE or quasi-TM operation around $1550 \mathrm{~nm}$ wavelength, given a fixed metal cladding separation.

\section{1D ANALYSIS}

We choose commonly available materials, namely silver (Ag), silica $\left(\mathrm{SiO}_{2}\right)$, and silicon $(\mathrm{Si})$, for the current theoretical study. Throughout this paper, we have explicitly considered the material dispersion. The dielectric constant of silver is described in Drude model as

$$
\epsilon(\mathrm{Ag})=\epsilon_{\infty}-\frac{\left(\epsilon_{0}-\epsilon_{\infty}\right) \omega_{p}^{2}}{\omega^{2}+i \omega \gamma}
$$

with $\epsilon_{\infty}=4.017, \epsilon_{0}=4.896, \omega_{p}=1.419 \times 10^{16} \mathrm{rad} / \mathrm{s}$, and $\gamma=1.117 \times 10^{14} \mathrm{rad} / \mathrm{s}$. This Drude model is fitted according to the measured data from Palik's handbook [10]. The dielectric constant of silica is assumed to adhere to the wellrecognized Sellmeier equation [11]. And similarly, a Sellmeier equation for silicon [10] is adopted. The operating wavelength is chosen to be within the range $1.2 \sim 2.0 \mu \mathrm{m}$, in which both silica and silicon are transparent.

We have employed a transfer-matrix method (TMM) for deriving modes propagating in the $1 \mathrm{D}$ waveguide. A time dependence of $\exp (j \omega t)$ is assumed throughout this study for harmonic electromagnetic field. The guided modes in a 1D waveguide can be separated into TE and TM two groups. We describe briefly the method for deriving TM modes. TE probelms can be handled accordingly. As a mode field in any single layer fulfills the wave equation in homogeneous material, and its general solution for the tangential field $\mathrm{H}_{y}$ can be written as

$$
\mathrm{H}_{y, i}=A \exp \left(j k_{x i} x\right)+B \exp \left(-j k_{x i} x\right)
$$

where $k_{x i}^{2}=k_{0}^{2} \mu_{r i} \epsilon_{r i}-\beta^{2}$. Care should be taken for choosing the sign of the square root when deducing $k_{x i} . k_{0}$ is the freespace wave number. $\beta$ is the propagation constant which is, in general, complex. The effective mode index is defined as $n_{\text {eff }}=\beta / k_{0}$. In (2), the first term denotes the left-propagating wave, and the second denotes the right-propagating wave. Another tangential field $\mathrm{E}_{z}$ can be derived as

$$
\mathrm{E}_{z i}=A \frac{k_{x i}}{\omega \epsilon_{i}} \exp \left(j k_{x i} x\right)-B \frac{k_{x i}}{\omega \epsilon_{i}} \exp \left(-j k_{x i} x\right) .
$$

Equations (2) and (3) can be written compactly as

$$
\left[\begin{array}{c}
\mathrm{H}_{y i} \\
\mathrm{E}_{z i}
\end{array}\right]=\mathbf{M}_{i}\left[\begin{array}{l}
A \\
B
\end{array}\right] .
$$

As tangential fields are continuous across an material interface, by setting $x=x_{i}$ with $x_{i}$ the $i$ th interface position, we can relate fields in two adjacent layers by

$$
\left[\begin{array}{c}
\mathrm{H}_{y i} \\
\mathrm{E}_{z i}
\end{array}\right]=\mathbf{M}_{x i}\left[\begin{array}{c}
\mathrm{H}_{y, i+1} \\
\mathrm{E}_{z, i+1}
\end{array}\right],
$$

where $\mathbf{M}_{x i}=\mathbf{M}_{i}^{-1} \mathbf{M}_{i+1}$ is the transfer matrix. In this manner, the field in the leftmost layer can be related to that in the rightmost layer. By considering there is no rightpropagating wave in the leftmost layer, and that there is no left-propagating wave in the rightmost layer, we can get a characteristic equation. $\beta$ values can be determined by solving for the roots of the equation.

Figures 2(a) and 2(b) show, respectively, the variations of the TE and TM modes in their $n_{\text {eff }}$ and loss values as the Si layer thickness $d$ is changed from 0 to $500 \mathrm{~nm}$ while the distance between two metals $w$ is kept at $500 \mathrm{~nm}$. Wavelength is at $1550 \mathrm{~nm}$. For the first TE mode, its propagation loss decreases sharply by more than one order and reaches its minimum at $25.7 \mathrm{~dB} / \mathrm{mm}$ around $d=220 \mathrm{~nm}$. The loss increases gently as $d$ is further increased. In fact, the $\mathrm{TE}_{0}$ mode is close to cutoff at $d=0 \mathrm{~nm}$, and introduction of the $\mathrm{Si}$ layer pushes the cutoff to longer wavelength, which explains the initial sharp decrease of loss. When $d$ reaches $275 \mathrm{~nm}$, the second TE mode $\left(\mathrm{TE}_{1}\right)$ begins to be guided. We can easily avoid this $\mathrm{TE}_{1}$ mode by letting $d<275 \mathrm{~nm}$. The TM modes in Figure 2(b), in general, exhibits the same trend. The first two modes, $\mathrm{TM}_{0}$ and $\mathrm{TM}_{1}$, are the Fano mode pair in a conventional MIM slab waveguide. Among them, the $\mathrm{TM}_{0}$ mode does not experience cutoff even if $w$ decreases to a nearzero value. Propagation loss of this fundamental mode decreases as $d$ increases. The minimum loss of $22.6 \mathrm{~dB} / \mathrm{mm}$ occurs when $d=340 \mathrm{~nm}$. This drop in loss is attributed to the trapping of light in the Si layer, and therefore the interaction of mode with metal surfaces is reduced. Notice that allSi filling results in a higher loss than that induced by all- $\mathrm{SiO}_{2}$ filling. The $\mathrm{TM}_{1}$ mode is close to cutoff at $d=0$, and it becomes better confined as the Si layer is introduced. However, the improvement is not as significant as that for the fundamental mode. The is due to the fact that there is a nodal line for the dominant field of the $\mathrm{TM}_{1}$ mode, which effectively expels light out of the Si layer. The $\mathrm{TM}_{2}$ mode appears at $d=275 \mathrm{~nm}$, which experiences similar dispersion and loss behaviors as the $\mathrm{TE}_{1}$ mode. The Fano mode pair is seen to always exist regardless of $d$. However, we will show in the next section that the second Fano mode can be stripped off if a proper substrate and superstrate are deployed for a realistic 2D waveguide. Here $w=500 \mathrm{~nm}$ has been chosen for our 

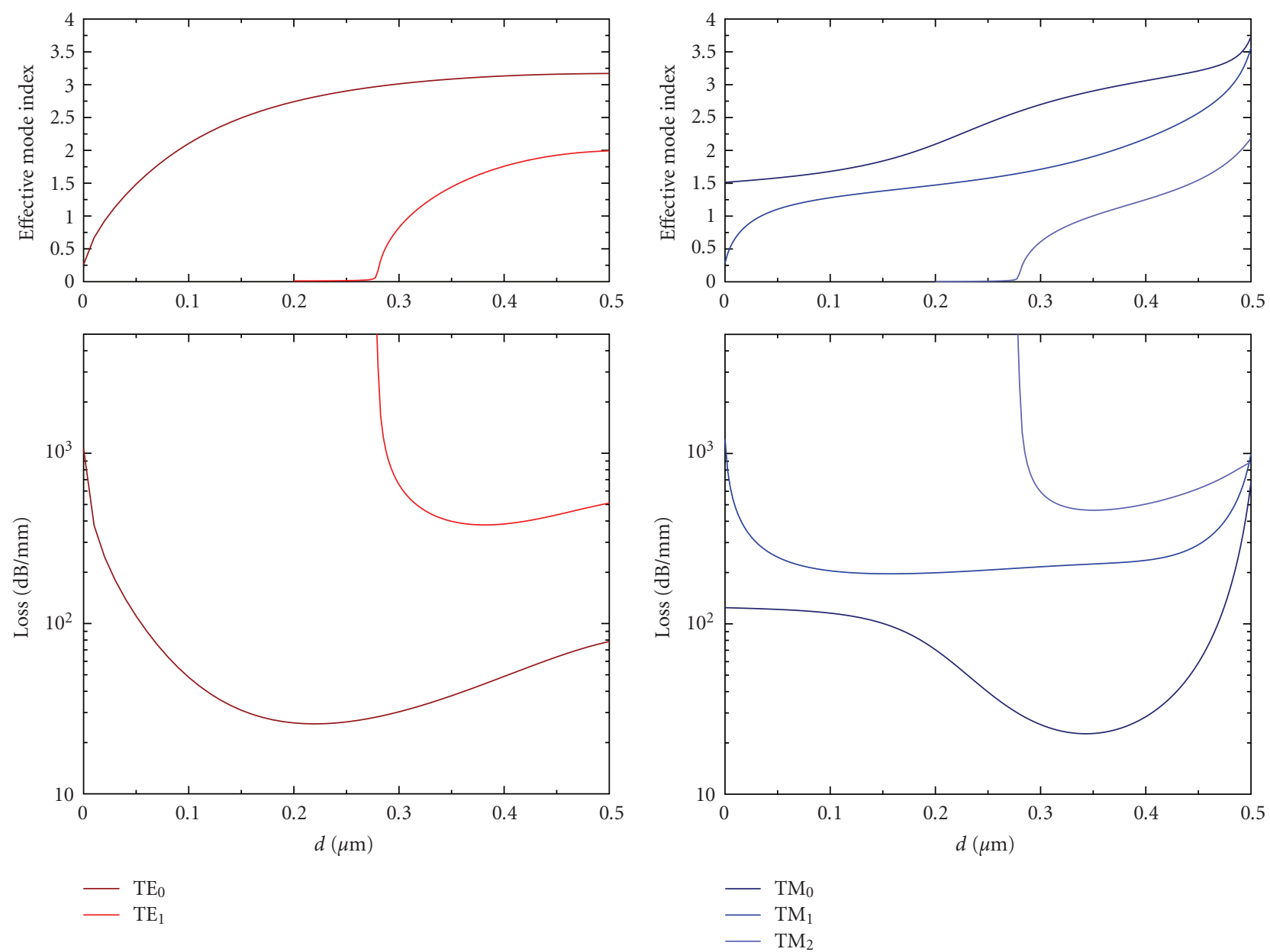

(a)

(b)

FIGURE 2: The variations of $n_{\text {eff }}$ (upper panel) and loss (lower panel) values for the TE (a) and TM (b) modes with respect to the Si layer thickness.
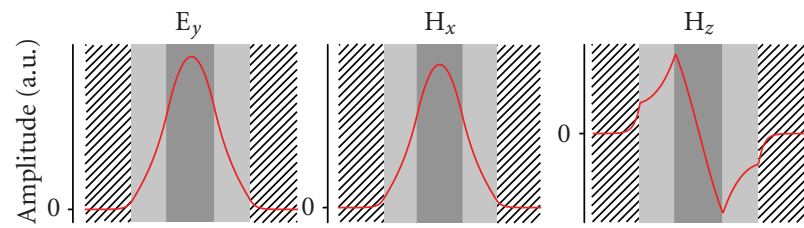

(a)
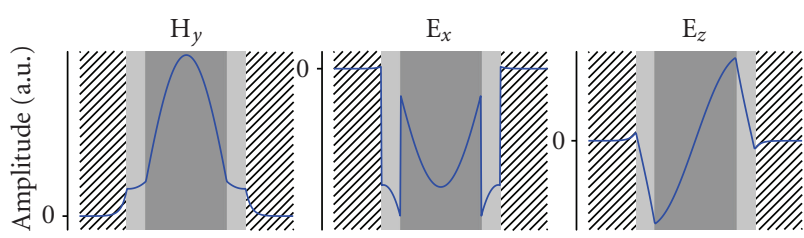

(b)

Figure 3: Modes supported by $1 \mathrm{D}$ waveguides. (a) Field components of the $\mathrm{TE}_{0}$ mode guided by a slab waveguide with $d=200 \mathrm{~nm}$. (b) Field components of the $\mathrm{TM}_{0}$ mode guided in a slab waveguide with $d=340 \mathrm{~nm}$. In both cases, the $z$ field component is the minor component. case study. Although not shown in this paper, we point out that an extra Si layer only helps to reduce propagation loss when $w$ is larger than $\sim 250 \mathrm{~nm}$. When $w=200 \mathrm{~nm}$, no TE mode is supported, and loss of the first TM mode increases monototically with $d$.

In Figure 3(a), we plot the fundamental TE mode at $d=200 \mathrm{~nm}$, and the fundamental TM mode at $d=340 \mathrm{~nm}$ in Figure 3(b). It is evident that a major part of the light is trapped in the Si layer, which reduces the interaction of the modes with the metal claddings. The field penetration into metal claddings is limited to only tens of nanometers (socalled skin depth). Therefore, it is expected that such waveguides can be placed very close to each other without suffering from heavy cross-talks, which is key for achieving highdensity optical integration.

\section{2D ANALYSIS}

In $2 \mathrm{D}$ scenario, the waveguide in Figure 1(a) will have a finite height $h$. A substrate and a superstrate will be present below and above the waveguide, respectively, as shown in 


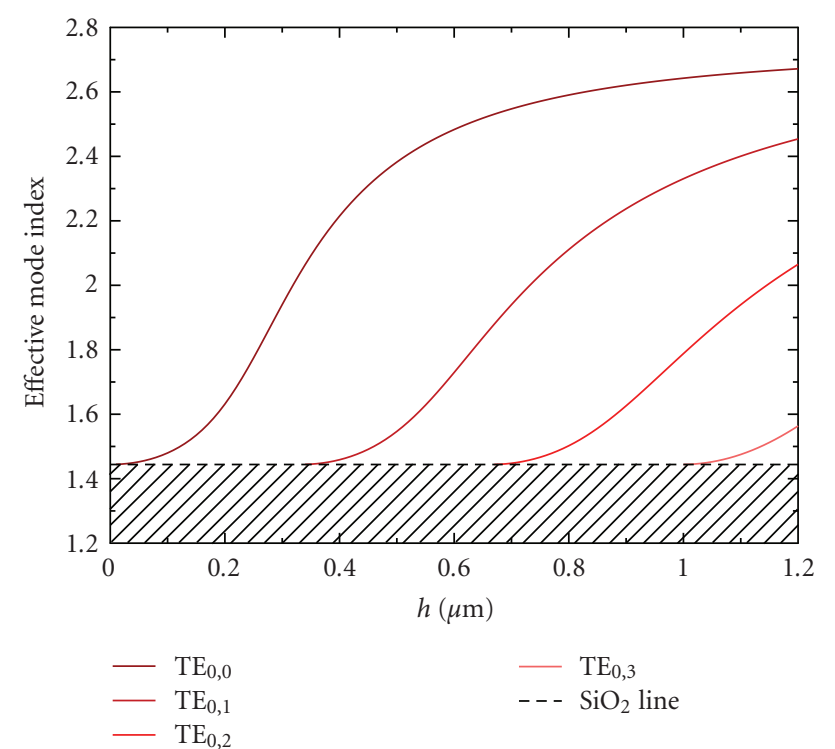

(a)

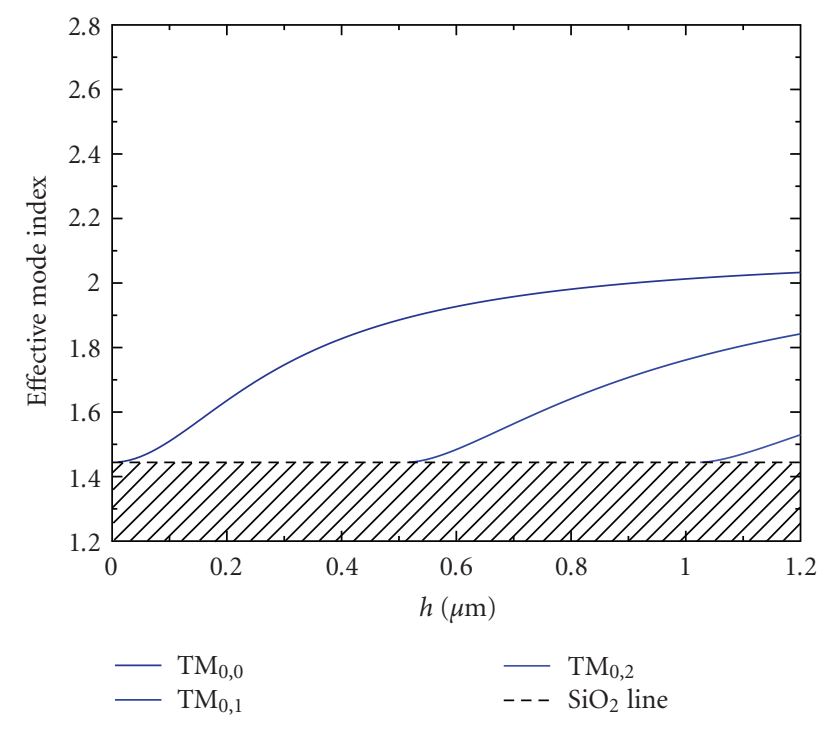

(b)

FIGURE 4: Dispersion of the TE (a) and TM (b) modes as the waveguide height is changed. $d=200 \mathrm{~nm}$.

Figure 1(b). The guided mode is affected not only by the height of the planar waveguide but also by the indices of substrate and superstrate. Most importantly, the high-order modes that appear in 1D waveguides as shown in Figure 2 can be trimmed off for some adequate substrate or superstrate. For example, it is relatively simple to achieve low-loss single-mode quasi-TE operation at $\lambda=1550 \mathrm{~nm}$, since a substrate and a superstrate made of $\mathrm{SiO}_{2}(n \approx 1.444)$ would be sufficient to get rid of the $\mathrm{TE}_{1}$ mode at $d=200 \mathrm{~nm}$ (see Figure 2(a)). For low-loss single-mode quasi-TM operation a substrate and a superstrate of index greater than $\sim 2.0$ (but less than 2.75) can be used to get rid of the odd Fano mode $\left(\mathrm{TM}_{1}\right)$ while $d$ can be kept around $340 \mathrm{~nm}$ (see Figure 2(b)). In the following, we will introduce two separate waveguides that are, respectively, intended for low-loss quasi-TE and quasi-TM operations.

\subsection{Waveguide for quasi-TE operation}

For low-loss quasi-TE operation, we let both substrate and superstrate be silica. $d$ is fixed at $200 \mathrm{~nm}$. In $1 \mathrm{D}$ limit, the loss of the TE-mode will be as low as $26.1 \mathrm{~dB} / \mathrm{mm}$ at $1550 \mathrm{~nm}$ wavelength (see Figure 2(a)). A further complication is on choosing an appropriate height $h$ to prevent nodal line from appearing in the vertical direction. Although a vector finiteelement method (FEM) can be used to decide $h$ for singlemode operation, we have found the effective-index method (EIM) a much more efficient way to achieve the objective.

In EIM, a 2D planar waveguide is simplified to a horizontally-placed three-layer slab waveguide. For the particular waveguide shown in Figure 1(b), the upper and bottom layers of the corresponding simplified waveguide are simply $\mathrm{SiO}_{2}$. The central layer has a refractive index which is the $n_{\text {eff }}$ value of the mode guided by the waveguide shown in Figure 1(a)). The EIM is noticed to have a reasonable accuracy, especially when wavelength is small (not shown). Figure 4(a) shows the geometric dispersion curves for the first four TE modes as $h$ is varied at $\lambda=1550 \mathrm{~nm}$. We have only considered the $\mathrm{TE}_{0}$ mode in Figure $2(\mathrm{a})$. It is seen that all high-order modes approach cutoff when $h$ decreases. When $h$ is less than $\sim 400 \mathrm{~nm}$, only one mode, that is, $\mathrm{TE}_{0,0},{ }^{1}$ is supported. In the same manner, we have examined the geometric dispersions for the TM modes, as shown in Figure 4(b); and it is noticed that all high-order modes have been cutoff for $h<\sim 500 \mathrm{~nm}$. Again, only $\mathrm{TM}_{0}$ mode in Figure 2(b) is studied here. Although the second mode in Figure 2(b) $\left(\mathrm{TM}_{1}\right)$ has an $n_{\text {eff }}=1.472768$, which is slightly larger than the refractive index of the substrate/superstrate (1.444024), the effective index of all modes in 1D case will be reduced as the height of the waveguide is reduced. It will be shown in the following paragraph that $\mathrm{TM}_{1, x}$ modes will not be supported in our designed 2D waveguide by using an appropriate $h$.

Next, we use a vector FEM method to investigate explicitly a particular 2D planar waveguide that is optimized for low-loss quasi-TE operation. The advantages of FEM include its accurate definition of material boundaries as well as its adaptive meshing of the numerical domain. The waveguide under study has a height of $450 \mathrm{~nm}$. The dispersion and loss curves for the first four modes are plotted in Figures 5(a) and 5(b). It is seen that the high-order $\mathrm{TM}_{0,1}$ and $\mathrm{TE}_{0,1}$ modes approach to their cutoffs at $\lambda \approx 1380 \mathrm{~nm}$ and $\lambda \approx 1430 \mathrm{~nm}$, respectively. Hence the waveguide operates in single mode

\footnotetext{
${ }^{1}$ The first subscript in the mode designation corresponds to the number of nodal lines along $x$ direction, while the second subscript denotes the number of nodal lines along $y$ direction. Although the modes are all hybrid, for simplicity we still use the abbreviations "TE" and "TM."
} 


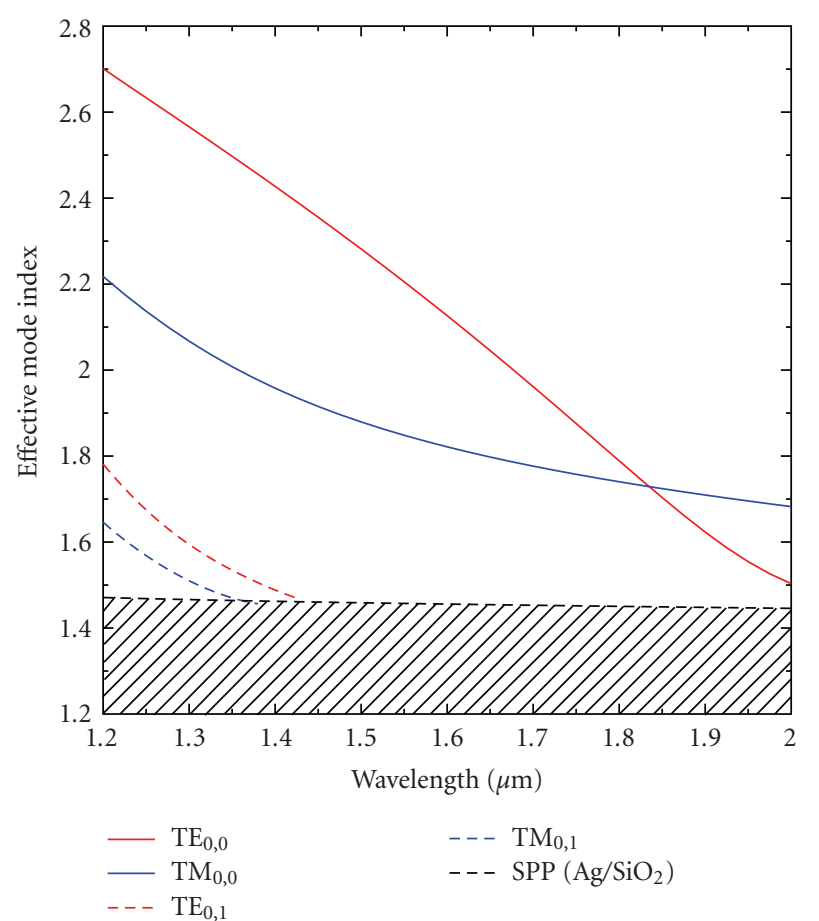

(a)

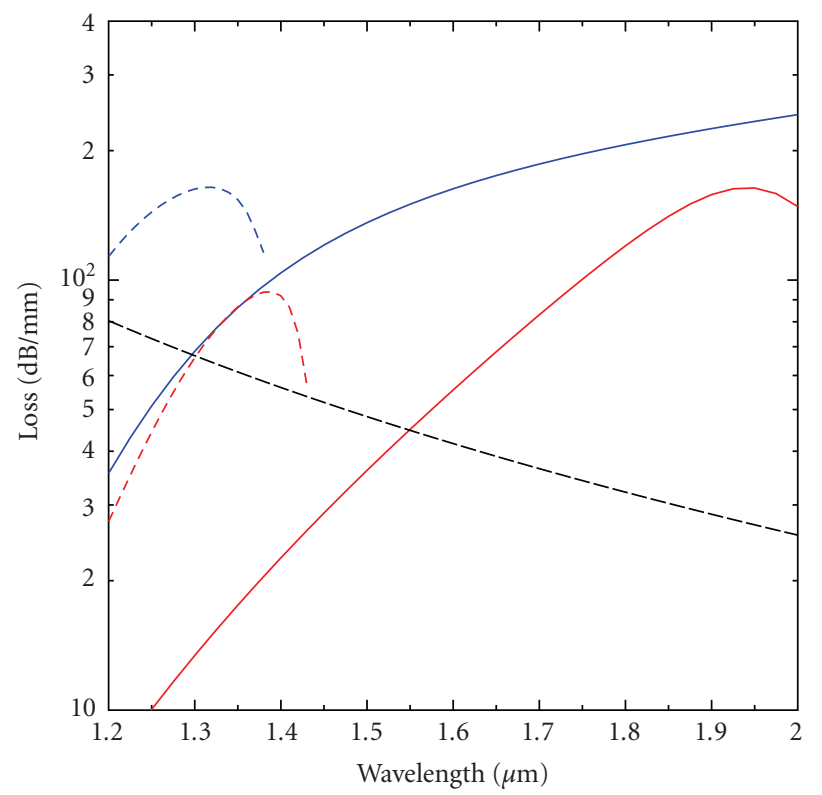

(b)

FIgURE 5: Dispersion (a) and loss (b) values for the first four modes guided by a $2 \mathrm{D}$ waveguide with $d=200 \mathrm{~nm}$ and $h=450 \mathrm{~nm}$.

for each polarization at wavelength beyond $\sim 1430 \mathrm{~nm}$. At $\lambda=1550 \mathrm{~nm}$, the waveguide is highly birefringent, with birefringence value (defined as $\left|n_{\text {eff }}^{x}-n_{\text {eff }}^{y}\right|$ ) as high as 0.3569 . This indicates that the $\mathrm{TM}_{0,0}$ and $\mathrm{TE}_{0,0}$ modes, though both supported by the waveguide, will hardly interact with each other. A careful excitation by a linearly polarized light source will ensure that only one of the modes will be propagating down the waveguide. Generally speaking, the propagation

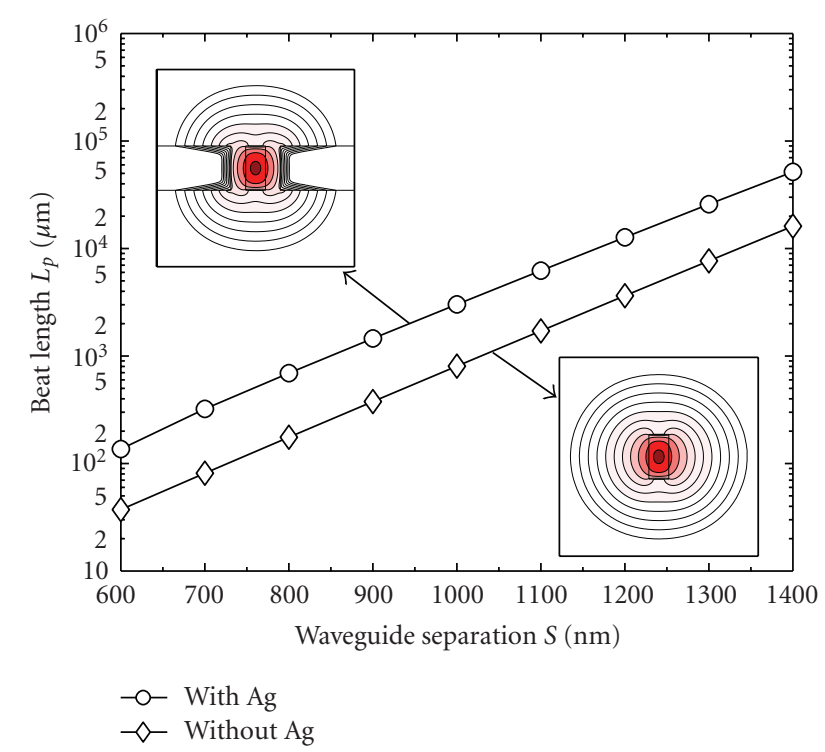

Figure 6: Beat length variation as a function of waveguide separation for the quasi-TE mode at $\lambda=1550 \mathrm{~nm}$. The inset in the upper-left corner gives the $\mathrm{TE}_{0,0}$ mode supported by an individual waveguide with metal cladding, while that in the bottom-right corner gives the same mode supported by a waveguide without metal cladding. The field contour lines are in $3 \mathrm{~dB}$ separation. The first contour line is an exception, which is at $90 \%$ of the maximum value. Domain size of the field plots: $2 \times 2 \mu \mathrm{m}^{2}$.

loss of the waveguide increases as $h$ is decreased. The propagation losses of both $\mathrm{TE}_{0,0}$ and $\mathrm{TM}_{0,0}$ modes are, in general, higher as compared to the pure $1 \mathrm{D}$ modes. The propagation loss of the $\mathrm{TE}_{0,0}$ mode at $1550 \mathrm{~nm}$ is $44.9 \mathrm{~dB} / \mathrm{mm}$, and that for $\mathrm{TM}_{0,1}$-like mode $150.1 \mathrm{~dB} / \mathrm{mm}$. The loss of the $\mathrm{TE}_{0,1}$-like mode is almost equal to that of the SPP mode supported by a single $\mathrm{Ag}-\mathrm{SiO}_{2}$ surface (see Figure 5(b)), which is in theory impossible to be achieved by the coupled SPPs in MIM waveguides.

To know how strong two waveguides placed in parallel interact with each other, one needs to examine the beat length $L_{p}$ for the coupled supermodes. Beat length is defined as $L_{p}=2 \pi /\left|\beta_{e}-\beta_{o}\right|$, where $\beta_{e}$ and $\beta_{o}$ are propagation constant of the even and odd supermodes, respectively. The longer the $L_{p}$, the weaker the two waveguides interact. In Figure 6, we show the variation of beat length as a function of the waveguide separation $S$ (defined as the distance between core center to core center). The same curve is also derived for an identical waveguide but without the metal claddings. Both curves are approximately linear with the beat length represented in logarithmic scale. It is observed that the beat length is increased to their 3.5 times when metal claddings are present. With the same cross-talk requirement, waveguides with metal claddings can be put $23 \%$ closer as compared to without metal cladding. According to the coupledmoded theory, field overlapping of two waveguide modes (examined for each individual waveguide in separation) has direct effect on the coupling strength between two waveguides. From the field distribution of the mode supported by the hybrid waveguide (upper-left inset in Figure 6), it is not 


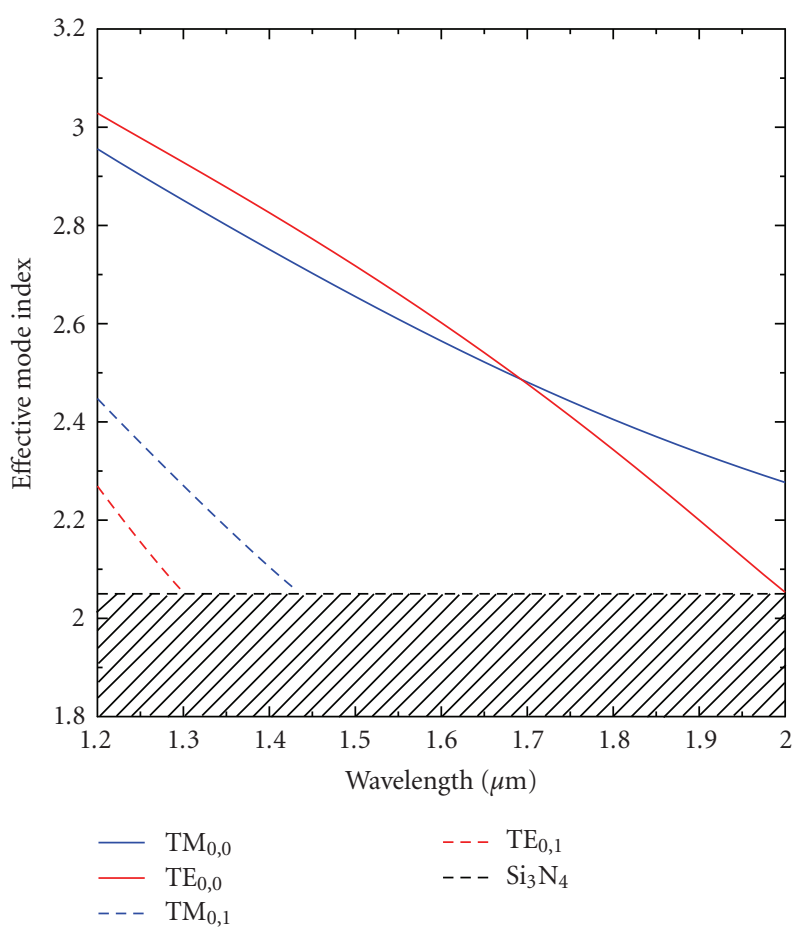

(a)

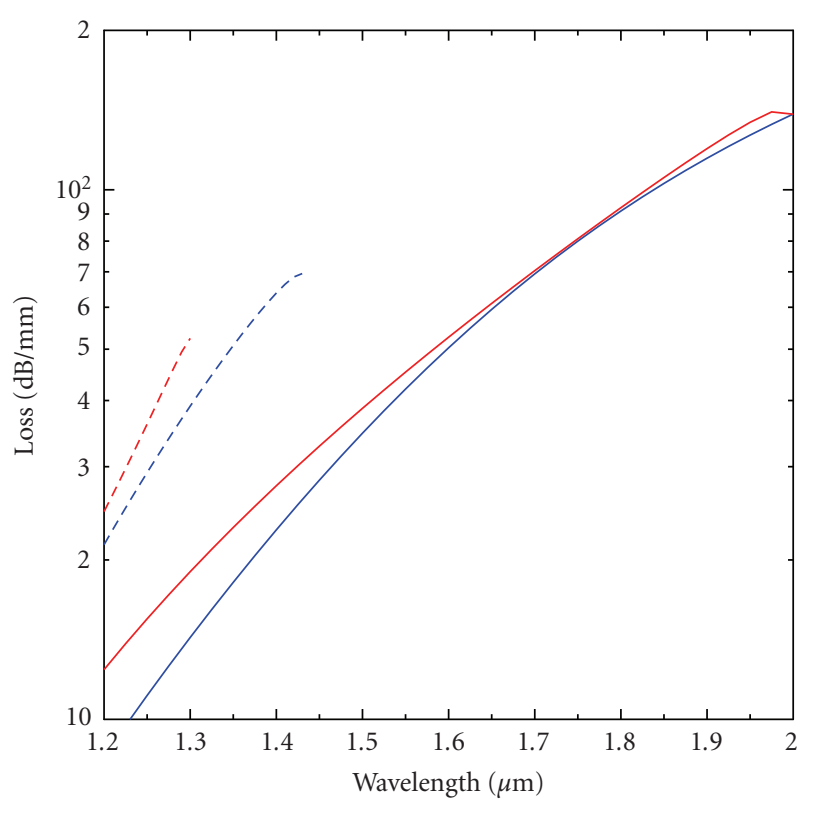

(b)

Figure 7: Dispersion (a) and loss (b) curves for guided modes in a waveguide with $d=340 \mathrm{~nm}$ and $h=480 \mathrm{~nm}$. $\lambda=1550 \mathrm{~nm}$.

difficult to understand that the field overlapping of such two modes will be smaller, as compared to waveguides without metal claddings.

\subsection{Waveguide for quasi-TM operation}

In this subsection, we look into waveguides that can operate with a relatively low-loss $\mathrm{TM}_{0,0}$ mode. We follow the previous sub-section by setting $w=500 \mathrm{~nm}$. To get rid of the odd Fano mode shown in Figure 2(b), we can choose Silicon Nitride $\left(\mathrm{Si}_{3} \mathrm{~N}_{4}\right.$, whose index is assumed to be 2.05 for $\lambda=1.2 \sim 2 \mu \mathrm{m}$ ) as both the substrate and the superstrate. The $\mathrm{Si}_{3} \mathrm{~N}_{4}$ layers are not placed in direct contact with the metals in order to avoid the high loss induced by $\mathrm{Ag}-\mathrm{Si}_{3} \mathrm{~N}_{4} \mathrm{SPP}$. In the present study, the $\mathrm{Si}_{3} \mathrm{~N}_{4}$ materials $200 \mathrm{~nm}$ away from the metal surfaces. See Figure 8 insets for the waveguide crosssection.

First, according to Figure 2(b), we choose $d=340 \mathrm{~nm}$ in order to minimize the absorption loss. Again, using EIM we estimate that the height $h$ of the core layer should be kept less than $500 \mathrm{~nm}$. In this investigation, we select $h=480 \mathrm{~nm}$. In Figure 7 , we show variation of the $n_{\text {eff }}$ and loss values with respect to wavelength. Similar to previously designed waveguide, the current waveguide supports both $\mathrm{TE}_{0,0}$ and $\mathrm{TM}_{0,0}$ two modes at $\lambda=1.55 \mu \mathrm{m}$. Losses of the two modes are comparable at $45.3 \mathrm{~dB} / \mathrm{mm}$ and $42.0 \mathrm{~dB} / \mathrm{mm}$, respectively. The birefringence at $\lambda=1.55 \mu \mathrm{m}$ is noticed to be 0.052 , which is smaller as compared to the value possessed by the waveguide shown in the previous subsection. Still, the birefringence is significantly larger than those in commercial HI-BI fibers, which are normally in the order of $10^{-4}$. Therefore, a carefully launched polarization state is likely to be preserved at the output end of the waveguide.

To examine the coupling strength between such two waveguides placed in parallel, we show the beat length $L_{p}$ as a function of their separation $S$ in Figure 8. The same curve for an identical waveguide but without the metal cladding is also superimposed. With the metal cladding, the beat length is increased to its 5.0 times at $S=600 \mathrm{~nm}$ and 10.7 times at $S=1400 \mathrm{~nm}$. At the same level of cross-talk requirement, the proposed hybrid waveguide can be placed up to $25 \%$ closer as compared to the waveguides without metal claddings (examined around $S=700 \mathrm{~nm}$ ). The major magnetic field $\mathrm{H}_{y}$ supported by an individual waveguide for both with and without metal claddings are shown as insets in Figure 8. Comparing the two fields, it can be seen that the mode guided by the hybrid waveguide is better confined laterally due to the presence of metal claddings. In fact, the major field components $\mathrm{H}_{y}$ and $\mathrm{E}_{x}$ are antiresonant with the horizontal $\mathrm{Ag}-\mathrm{SiO}_{2}$ interfaces; therefore, a quicker field decay along the surfaces is resulted.

\section{DISCUSSION AND CONCLUSION}

The losses of the designed waveguides are in the range of $40 \sim 50 \mathrm{~dB} / \mathrm{mm}$. Such a loss value allows a signal to be transmitted for a $\sim 100 \mu \mathrm{m}$ distance before its power is dropped to its $1 / e$. Since the cross-sections of the waveguides are around $0.5 \times 0.5 \mu \mathrm{m}^{2}$, the functioning waveguides have a length-tocross-section aspect ratio of $\sim 200$. This value is reasonably 


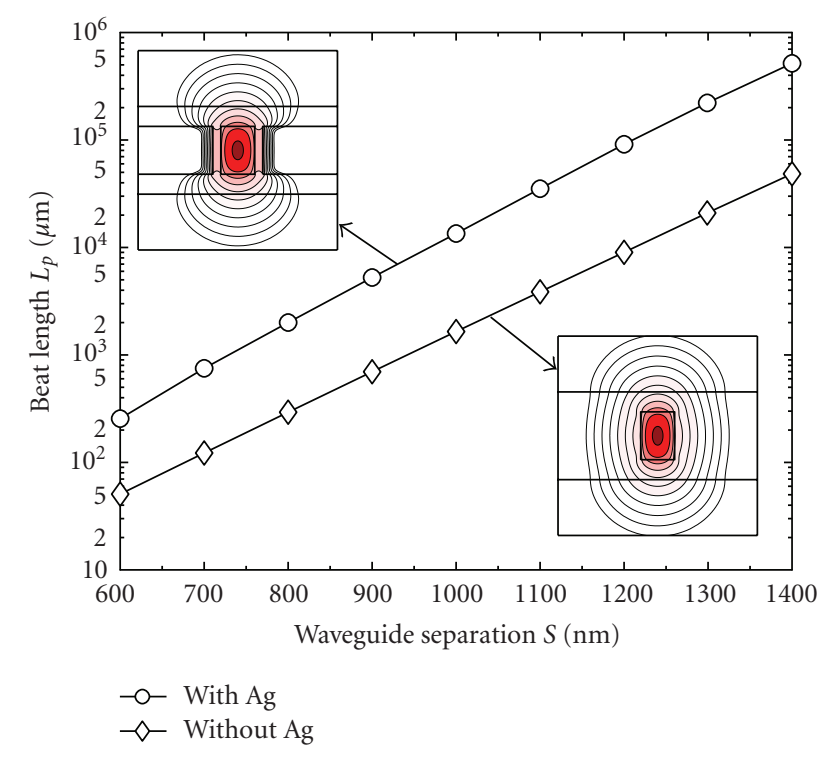

FIgURE 8: Beat length variation as a function of waveguide separation for the quasi-TE mode at $\lambda=1550 \mathrm{~nm}$. The inset in the upperleft corner gives the $\mathrm{TM}_{0,0}$ mode supported by an individual waveguide with metal cladding, while that in the bottom-right corner gives the mode supported by a waveguide without metal cladding. The field contour lines are in $3 \mathrm{~dB}$ separation. The first contour line is an exception, which is at $90 \%$ of the maximum value. Domain size of the field plots: $2 \times 2 \mu \mathrm{m}^{2}$.

large for achieving a wide varieties of photonic applications (e.g., ring resonators, couplers, etc.).

The designed waveguides have confirmed that it is possible to obtain both reasonably low-loss propagation as well as good confinement by combining both index-guiding and SPP-guiding mechanisms. There are several ways to achieve even lower loss, probably at the expense of field confinement. First, the propagation loss can be reduced by using some dielectric material with a lower epsilon value (e.g., air), in replacement of the $\mathrm{SiO}_{2}$ material in the proposed structures. A drop in propagation loss is expected because that the SPP guided by an Ag-air interface has a significantly lower loss than that guided by an $\mathrm{Ag}-\mathrm{SiO}_{2}$ interface. However, planar waveguides with their core exposed in air are relatively more vulnerable to environmental changes. Notably, contamination of water molecules will affect the guided modes significantly. Second, propagation loss can be drastically reduced if the gap size is allowed to increase to some higher value. Finally, waveguides with lower loss may be possible by applying the same design methodology to other waveguide structures, other than based on the MIM structure.

In conclusion, we have proposed the deployment of waveguides which propagate light by both index-guiding and SPP-guiding principles. With the hybrid guidance mechanism, it is possible to realize low-loss, compact-optical waveguides using commonly available materials. As a simple illustration, we have designed waveguides with their cross-sections at $\sim 500 \times 500 \mathrm{~nm}^{2}$ functioning around the $1550 \mathrm{~nm}$ telecommunication wavelength. The waveguides can be optimized for low-loss quasi-TE or quasi-TM guidance. An increase in packaging density by up to $25 \%$ is observed compared to using waveguides without metal claddings. The waveguide in quasi-TM operation deserves some special attention, since a down-tailoring of the waveguide does not lead to a cutoff of the guided quasi-TM mode, even when the waveguide's high-index inner core ceases to exist. We are currently exploring the possibility of coupling light efficiently from a waveguide proposed in this paper to a deep-subwavelength metal-gap SPP waveguide without an inner dielectric core though such a tailoring.

\section{ACKNOWLEDGMENTS}

This work is supported by the Swedish Foundation for Strategic Research (SSF) through the INGVAR program, the SSF Strategic Research Center in Photonics, and the Swedish Research Council (VR).

\section{REFERENCES}

[1] H. A. Atwater, "The promise of plasmonics," Scientific American, vol. 296, no. 4, pp. 56-63, 2007.

[2] P. Berini, "Figures of merit for surface plasmon waveguides," Optics Express, vol. 14, no. 26, pp. 13030-13042, 2006.

[3] M. Yan and M. Qiu, "Guided plasmon polariton at 2D metal corners," Journal of the Optical Society of America. B, Optical Physics, vol. 24, no. 9, pp. 2333-2342, 2007.

[4] L. Liu, Z. Han, and S. He, "Novel surface plasmon waveguide for high integration," Optics Express, vol. 13, no. 17, pp. 66456650, 2005.

[5] F. Kusunoki, T. Yotsuya, J. Takahara, and T. Kobayashi, "Propagation properties of guided waves in index-guided two-dimensional optical waveguides," Applied Physics Letters, vol. 86, no. 21, Article ID 211101, 3 pages, 2005.

[6] J. A. Dionne, L. A. Sweatlock, H. A. Atwater, and A. Polman, "Plasmon slot waveguides: towards chip-scale propagation with subwavelength-scale localization," Physical Review B-Condensed Matter and Materials Physics, vol. 73, no. 3, Article ID 035407, 9 pages, 2006.

[7] G. Veronis and S. Fan, "Guided subwavelength plasmonic mode supported by a slot in a thin metal film," Optics Letters, vol. 30, no. 24, pp. 3359-3361, 2005.

[8] D. F. P. Pile, T. Ogawa, D. K. Gramotnev, et al., "Twodimensionally localized modes of a nanoscale gap plasmon waveguide," Applied Physics Letters, vol. 87, no. 26, Article ID 261114, 3 pages, 2005.

[9] L. Chen, J. Shakya, and M. Lipson, "Subwavelength confinement in an integrated metal slot waveguide on silicon," Optics Letters, vol. 31, no. 14, pp. 2133-2135, 2006.

[10] E. D. Palik, Handbook of Optical Constants of Solids, Academic Press, Boston, Mass, USA, 1985.

[11] G. Agrawal, Nonlinear Fiber Optics, Academic Press, Boston, Mass, USA, 3rd edition, 2001. 

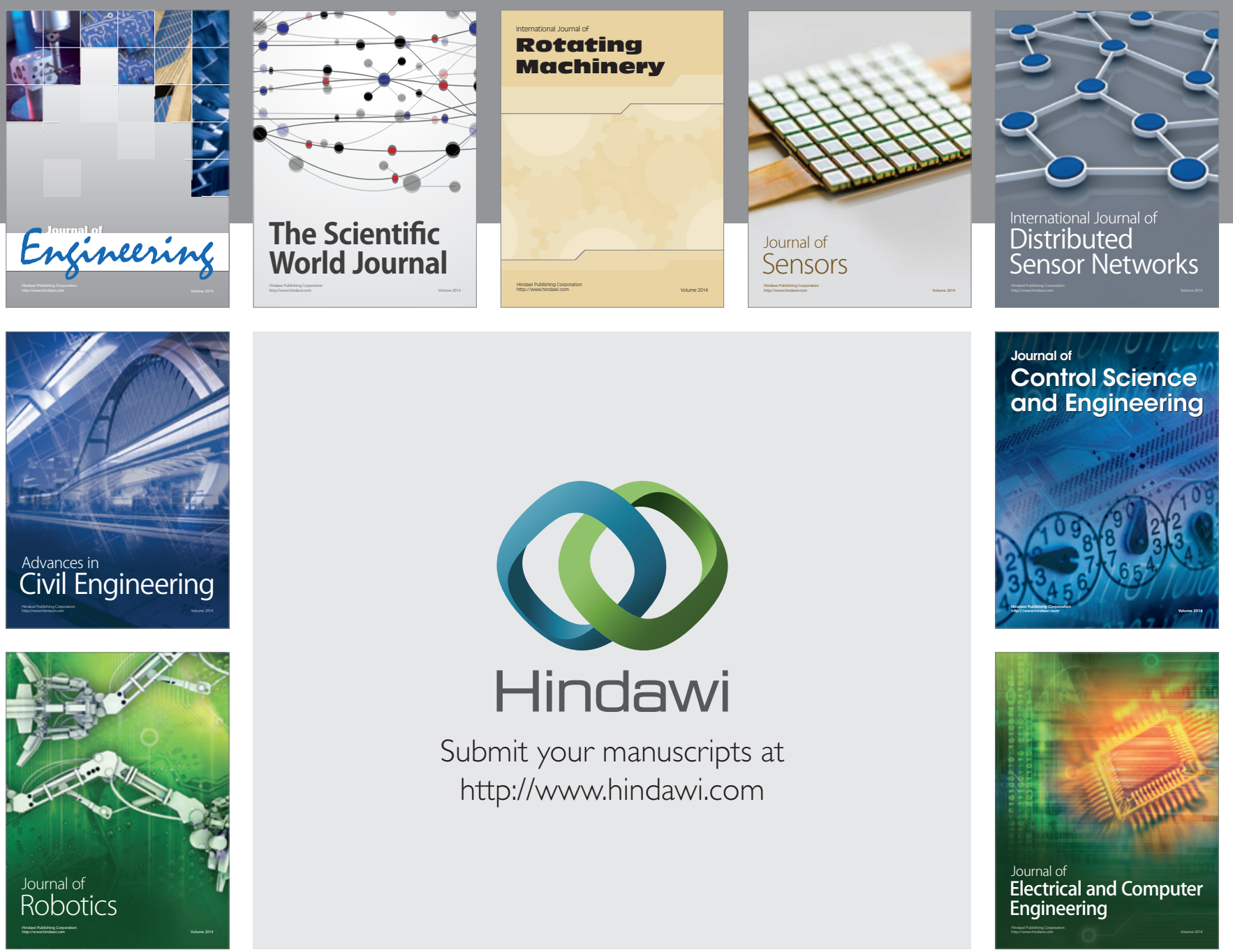

Submit your manuscripts at

http://www.hindawi.com
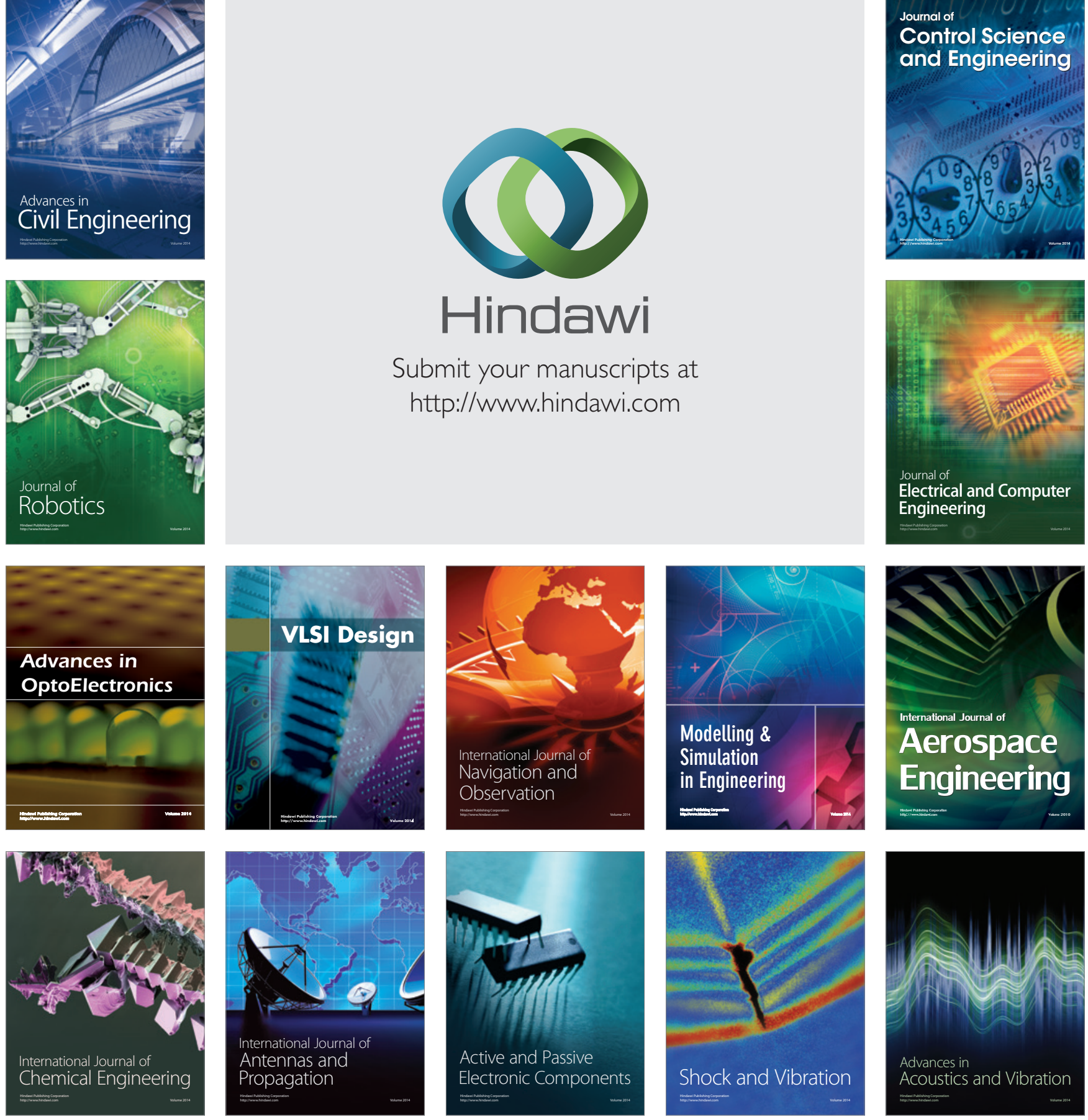